\title{
Tendencias en la paridad de género en carreras universitarias de computación en Chile: 2004-2018
}

\author{
Karla Soria-Barreto y Sergio Zuniga-Jara \\ Universidad Católica del Norte, Escuela de Ciencias Empresariales. Larrondo 1281, Coquimbo-Chile. \\ (correo-e: ksoria@ucn.cl; sz@ucn.cl)
}

Recibido Dic. 20, 2019; Aceptado Feb. 15, 2020; Versión final Abr. 8, 2020, Publicado Oct. 2020

\begin{abstract}
Resumen
Este artículo analiza la paridad de género en las carreras universitarias del área de computación que están adscritas al Sistema Único de Admisión (SUA) en Chile. El análisis es de tipo descriptivo. Los resultados muestran que en el año 2018 se matricularon un total de 5.381 mujeres (19,2\% del total). En las carreras de ingeniería civil ofrecidas por la Universidad de Chile, la Pontificia Universidad Católica, la Universidad Adolfo Ibáñez y la Universidad de Los Andes llegaron a un ingreso del 27\% de mujeres en el año 2018. Sin embargo, en las carreras de ingeniería civil (computación) ofrecidas por las demás universidades chilenas la paridad de género cayó del $17 \%$ en el año 2014 al $11 \%$ en el año 2018 . Se concluye que los programas pro-paridad de género como los implementados por la Universidad de Chile para las ingenierías parecen convenientes si se quiere incrementar la participación de las mujeres.
\end{abstract}

Palabras clave: ingeniería computación; paridad de género; proceso admisión; mujeres; Chile

\section{Trends in gender parity in computer science degrees in Chile: 2004-2018}

\begin{abstract}
This article analyzes gender parity in the computer science degrees that are registered in the Chilean Single Admission System (SAS). The database analysis is descriptive. The results show that $5,381(19.2 \%)$ female students enrolled in the computer science programs in 2018. There is a $27 \%$ female enrollment in 2018 in the civil engineering degrees offered by the Universidad de Chile, the Pontificia Universidad Católica, the Universidad Adolfo lbáñez, and the Universidad de Los Andes. However, gender parity fell from $17 \%$ in 2014 to $11 \%$ in 2018 in the civil engineering degrees (computer science) offered by other Chilean universities. It is concluded that pro-equity gender programs such as those implemented by the Universidad de Chile for engineering degrees seem to be appropriate to increase female enrollment.
\end{abstract}




\section{INTRODUCCIÓN}

Para referirse a diferencias de género que pueden existir en cualquier ámbito se suele referir a equidad (equity), igualdad (equality) y paridad (parity). Mientras que la equidad es dar a todos lo que necesitan para tener éxito, la igualdad consiste en tratar a todos por igual. Por el contrario, la paridad es una medida del acceso relativo de varones y mujeres a diferentes campos, tales como la educación, el trabajo o la representación popular (Mullally, 2007). En esta investigación nos enfocamos en mediciones de la paridad de género en algunas carreras universitarias de Chile. El proceso de transformación digital que está teniendo el mundo hace que cada vez se requieran más profesionales del área de las tecnologías, incluyendo las ciencias de la computación (Mora-Rivera et al., 2017). Las carreras vinculadas a la tecnología ofrecen buenos salarios y son una fuente de movilidad social. Por lo tanto, sería deseable tener una cantidad cada vez mayor de jóvenes de ambos sexos incorporándose en esta área del conocimiento. Según Pineda y González (2016), de acuerdo a datos del año 2019, en Chile ya existe un déficit del $31 \%$ de profesionales en tecnologías de información, cifra que representa unas 19.000 vacantes.

Sin embargo, la evidencia muestra que la participación de las mujeres en las carreras vinculadas con las disciplinas STEM (siglas en inglés que incluye a las ciencias básicas, tecnología, ingeniería y matemáticas) va en retroceso. Esto ha sido observado tanto en Chile, en los países de la OECD (ComunidadMujer, 2017) y a través del todo en el mundo (Katz, et al., 2006; Vilner y Zur, 2006; Williams y Emerson, 2002). En efecto, aunque el número absoluto de mujeres en esta área ha ido aumentando, su proporción respecto de los hombres es cada vez menor (Kesar, 2018). Las causas que se han considerado son diversas. Se sugiere que los hombres tienen un mayor interés temprano por las computadoras (Arango, 2006). También, diferencias en capacidades individuales, procesos de construcción de la identidad profesional (Hawes y Corvalán, 2005), y finalmente estereotipos sociales de género vinculados a la autoestima, ego y propósito de ser (ÁlvarezAguilar et al., 2019). El problema es complejo, ya que en muchos casos existe una orientación familiar que se da en la infancia de las niñas, es decir estereotipos sociales. También es posible que al interior de las aulas de educación básica y secundaria los procesos educativos sean distintos para hombres y mujeres. En adición, la vida profesional de hombres y mujeres después del grado puede ser bastante distinta, y las diferencias no se agotan en la universidad (Blázquez et al., 2012).

Las carreras de ingeniería y ciencias son consideradas poco femeninas por nuestra sociedad, gran porcentaje de las escuelas, liceos y colegios no estimulan en las niñas el gusto por las matemáticas y la física. Este fenómeno no es exclusivo de Chile, sino mundial y se ha mantenido a lo largo de la historia de la humanidad (García, 2004 y Ordorika, 2015). Pareciera que se ha estereotipado ciertas disciplinas educacional y ocupacionalmente, categorizándolas como masculinas (Anker, 1997). El tema de los estereotipos aparece desde la educación escolar, y es un problema a nivel mundial. Por ejemplo, para 16 países de la OECD los resultados de la prueba PISA 2015 de ciencias y matemáticas muestra que existen brechas significativas a favor de los hombres en puntajes de ciencias y matemáticas (ComunidadMujer, 2017).

En principio, el sistema educativo chileno no presenta brechas de género en el acceso a la educación. El problema tampoco está en la matrícula de mujeres a la educación superior de Chile, ya que según datos oficiales del Departamento de Evaluación, Medición y Registro Educacional (DEMRE, 2019), el porcentaje de mujeres matriculadas supera levemente al de los hombres. Sin embargo, en las áreas del conocimiento vinculadas a las disciplinas STEM, a situación es muy diferente, con una alta concentración de varones (Rolando et al., 2010). respecto de los países de la OECD, la proporción de mujeres tituladas en las carreras STEM ubican a Chile en el último lugar, con sólo un 19\%, en comparación a Polonia, por ejemplo, con un 44\% (ComunidadMujer, 2017). Para el año 2018 en Chile sólo uno de cada cuatro matriculados en las áreas STEM es mujer. Asimismo, una de cada cinco matrículas en tecnología son mujeres, y sólo un $5 \%$ de las mujeres trabajan en áreas de tecnológicas (Pineda y González, 2016). La proporción de mujeres que ingresan a estudiar alguna ingeniería civil (o plan común) es tan solo del $20 \%$ cada año, independiente de la universidad.

A pesar de su importancia, en Chile existe escasa literatura acerca de la evaluación de la participación de las mujeres en carreras universitarias en las áreas de computación. En Australia, Downes, et al. (2000) encuentran proporciones de participación femenina razonables en estas carreras, mientras en España, Merelo y Merelo (2017) observan una fuerte reducción en este porcentaje en los estudios universitarios de informática y en carreras científicas o técnicas. En México, Oliveros et al., (2016) reportan que la matrícula en ingeniería de las mujeres en México ha tenido un crecimiento lento, y sólo el $30 \%$ son mujeres. En Noruega, Pappas et al., (2016) encontraron diferencias de género (para las ganancias cognitivas, el compromiso afectivo, la motivación para estudiar y la satisfacción en carreras de ciencias de la informática). González (2014) reporta que el discurso sigue justificando la cultura masculinizada de las carreras tecnológicas y de ingenierías. Machina y Gokhale (2015) reportan que los blogs y sesiones con profesionales son herramientas efectivas para reclutar estudiantes mujeres para que elijan cursos del área de informática. 
En resumen, la literatura muestra que el estudio de las diferencias en la participación de mujeres y hombres en áreas de computación es creciente, y que actualmente es poco lo que se sabe en Chile. Una forma de avanzar en el estudio de este fenómeno en Chile es analizar los resultados de los procesos de admisión a las universidades chilenas en estas áreas, buscando identificar disparidades de género como primer paso para establecer políticas que permitan limitar las causas a todos los niveles. El aporte de este estudio está en proporcionar un análisis de las principales tendencias en las carreras universitarias del área de computación en Chile, con foco en las diferencias entre hombres y mujeres. El trabajo se organiza presentando en primer lugar la metodología, luego los resultados, y se finaliza con la discusión y conclusiones.

\section{METODOLOGÍA}

La información primaria de este estudio proviene de las bases de datos de los procesos de admisión del Sistema Único de Admisión (SUA) chileno entre los años 2004 y 2018, los que fueron proporcionados por el Departamento de Evaluación, Medición y Registro Educacional (DEMRE, 2019). La Tabla 1 muestra la forma en que este sistema de admisión ha ido ampliándose desde 25 universidades integrantes en el periodo 20042011, hasta 41 universidades el año 2018. Se considera aquí solamente la información de la admisión vía ingreso directo de todas las universidades indicadas en la Tabla. La base de datos quedó conformada por un total de 3.389.084 registros, correspondientes a los estudiantes inscritos que rindieron la Prueba de Selección Universitaria (PSU) en el periodo 2004-2018 y que postularon a un total de 66 programas ofrecidos por las 41 universidades pertenecientes al SUA.

Tabla 1: Evolución del número de universidades chilenas adscritas al Sistema Único de Admisión (SUA)

\begin{tabular}{|l|c|l|}
\hline Año & $\begin{array}{l}\text { Número de Universidades } \\
\text { Adscritas }\end{array}$ & Universidades \\
\hline $2004-2011$ & 25 & CRUCH \\
\hline $2012-2015$ & 33 & CRUCH; UDP; UMAYOR; UFT; UNAB; UAI; UANDES; UDD; UAH \\
\hline 2016 & 36 & $\begin{array}{l}\text { CRUCH (UOH; UAYSEN); UDP; UMAYOR; UFT; UNAB; UAI; } \\
\text { UANDES; UDD; UAH; UCSH }\end{array}$ \\
\hline 2017 & 39 & $\begin{array}{l}\text { CRUCH (UOH; UAYSEN); UDP; UMAYOR; UFT; UNAB; UAI; } \\
\text { UANDES; UDD; UAH; UCSH; UA; USS; UCENTRAL }\end{array}$ \\
\hline 2018 & 41 & $\begin{array}{l}\text { CRUCH (UOH; UAYSEN); UDP; UMAYOR; UFT; UNAB; UAI; } \\
\text { UANDES; UDD; UAH; UCSH; UA; USS; UCENTRAL; UAHC; UBO }\end{array}$ \\
\hline
\end{tabular}

El CRUCH es el llamado Consejo de Rectores de Universidades Chilenas, y lo integran las siguientes universidades: U. de Chile; Pontificia U. Católica de Chile; U. de Concepción; Pontificia U. Católica de Valparaíso; U. Técnica Federico Santa María; U. de Santiago de Chile; U. Austral de Chile; U. Católica del Norte; U. de Valparaíso; U. de Antofagasta; U. de La Serena; U. de La Frontera; U. de Magallanes; U. de Talca; U. de Atacama; U. de Tarapacá; U. Diego Portales; U. Arturo Prat; U. Metropolitana de Ciencias de la Educación; U. de Playa Ancha; U. del Bío-Bío; U. de Los Lagos; U. Tecnológica Metropolitana; U. Católica del Maule; U. Cat. de la Santísima Concepción; U. Católica de Temuco; U. Alberto Hurtado; U. de O'Higgins y U. de Aysén.

Para identificar la oferta de carreras universitarias del área de computación e informática se realizaron varios ajustes a la base de datos, seleccionando solamente las carreras que tuvieran como nombre alguna de las palabras "computación" o "informática". No fueron incluidas las carreras que se relacionaban indirectamente al área, como es el caso de la Licenciatura en Física mención Computación Científica. Tampoco se consideraron las carreras que ofrecieron vacantes, pero no obtuvieron suficientes matriculados, como es el caso, por ejemplo, de Ingeniería en Ejecución Informática modalidad vespertina de la U. de Tarapacá en el 2014. En la preparación de la base de datos se ajustaron varias situaciones tales como los cambios de nombre de carreras, y los cambios de código DEMRE en un año, pero con demás datos idénticos (se asumió que se trataba de la misma carrera).

Debido al gran número de programas-carreras ofrecidas en computación e informática, éstas fueron organizadas en siete grupos principales, según el título profesional que entregan. La Tabla 2 muestra que el Grupo 0 se refiere a la admisión a la carrera de Ingeniería Civil plan común. Se incluye en algunos casos el código que usa el SUA para identificar cada carrera. Para este grupo no se cuenta con información exacta del número de estudiantes que, luego de cursar el plan común de 2 años, finalmente optó por el área de computación. Se trata de sólo 4 universidades: U. de Chile, Pontificia U. Católica de Chile, U. de Los Andes y U. Adolfo lbáñez. En el caso del Grupo 1, incluye las carreras de ingeniería que tienen ingreso directo: Ing. Civil Bioinformática; Ing. Civil Computación; Ing. Civil Computación e Informática; Ing. Civil Computación mención Informática; Ing. Civil Industrial m. Informática; Ing. Civil Informática; Ing. Civil Informática y Telecomunicaciones; Ing. Civil Informática y Telecomunicaciones; Ing. Civil Informática-Lic. en Ciencias de la Ingeniería. En el Grupo 2 se incluye: Ing. Bioinformática; Ing. Computación; Ing. Computación e Informática 
(diurno y vespertino); Ing. Computación, Licenciatura en Ciencias de la Computación; Ing. Gestión Informática; Ing. Informática; Ing. Informática c/m en Gestión de la Inf., Lic. en Cs. de la Ing.; Ing. Informática c/m en Gestión de la Inf.; Ing. Informática Empresarial. El Grupo 3 contiene: Ing. Ejec. Computación e Informática; Ing. Ejec. Computación e Informática y Ing. Ejec. Informática. El Grupo 4 incluye: Tec. Universitario en Informática y Tecnólogo en Informática. El Grupo 5: Ped. Educación Media en Matemática y Computación: Ped. Matemática e Informática Educativa; Ped. Matemática y Computación; Ped. Matemática y Computación / Lic en Educ Mat y Computación; Ped. Matemática y Computación, Licenciatura en Educación. Finalmente, el Grupo 6: Lic. en Ciencia de la Computación.

Tabla 2: Los siete grupos de carreras de computación ofrecidas en Chile

\begin{tabular}{|c|c|c|c|c|}
\hline $\begin{array}{l}\text { Nombre de la } \\
\text { carrera }\end{array}$ & Grupo & Observaciones & $\begin{array}{l}\text { Vacantes } \\
2018\end{array}$ & $\begin{array}{l}\text { Matrícula } \\
2018\end{array}$ \\
\hline $\begin{array}{l}\text { Ingeniería Civil, } \\
\text { plan común }\end{array}$ & 0 & $\begin{array}{l}\text { Universidades que ofrecen acceso indirecto: } \\
\text { a) U. de Chile (11045 Ingeniería y Ciencias - plan común; } \\
\text { Santiago) } \\
\text { b) PUC (12039 Ingeniería; Santiago) } \\
\text { c) UAl ( } 42002 \text { Ingeniería Civil, plan común; Santiago, y } 42022 \\
\text { Ingeniería Civil, plan común; Viña del Mar) } \\
\text { d) U. Andes (43013 ingeniería civil (plan común); Santiago) }\end{array}$ & & \\
\hline Ingeniería Civil & 1 & $\begin{array}{l}\text { Universidades que ofrecen acceso directo: Este grupo es } \\
\text { ofrecido por } 25 \text { universidades }\end{array}$ & 2191 & 2104 \\
\hline $\begin{array}{l}\text { Ingeniería (no } \\
\text { Civil) }\end{array}$ & 2 & Ofrecido por 8 universidades & 662 & 477 \\
\hline $\begin{array}{l}\text { Ingeniería en } \\
\text { Ejecución }\end{array}$ & 3 & Ofrecido por 4 universidades & 225 & 237 \\
\hline $\begin{array}{l}\text { Técnico } \\
\text { Universitario }\end{array}$ & 4 & Ofrecido por 1 universidad (UTFSM) & 110 & 112 \\
\hline Pedagogía & 5 & Ofrecido por 7 universidades & 251 & 258 \\
\hline Licenciatura & 6 & Ofrecido por 1 universidad (USACH) & 45 & 46 \\
\hline
\end{tabular}

El análisis de las bases de datos es del tipo descriptivo, y se analizan las tendencias recientes en paridad de género de los estudiantes inscritos para rendir la PSU, y la paridad en el ingreso a cualquier universidad chilena. Luego se analizan comparativamente los puntajes promedio obtenidos en la PSU, tanto por género como por tipo de colegio de enseñanza media del cual egresaron los estudiantes. Luego se analiza comparativamente la paridad de género de los estudiantes que efectivamente se matriculan en carreras de computación en Chile, para cada uno de los grupos de carreras indicadas en la Tabla 1. Finalmente se revisan los aspectos referidos a la disposición de estudiantes hombres y mujeres para estudiar este tipo de carreras universitarias en regiones diferentes de su residencia habitual.

\section{RESULTADOS Y DISCUSIÓN}

A continuación se detallan cada uno de los resultados obtenidos. Estos presentan su evolución a lo largo del periodo de análisis.

\section{Paridad de género en el número de Inscritos para rendir la PSU}

La Figura 1 muestra que año tras año son más mujeres que hombres los que se inscriben para rendir la PSU. En general la cantidad de inscritos exhibe una tendencia creciente, y no se observan importantes variaciones en la composición estructural año tras año por sexo. En los 15 años analizados, la proporción promedio de mujeres inscritas es de $53 \%$ versus $47 \%$ de hombres. Esto significa que anualmente se inscriben para rendir la PSU unas 13.580 mujeres por sobre los inscritos varones. La principal causa de esta diferencia no es demográfica, ya que en Chile existe una leve mayor proporción de hombres que de mujeres en edades inferiores a los 19 años. Más bien causas económicas y culturales podrían empujar a los varones a estudiar carreras más cortas (no universitarias) e ingresar más rápidamente al mercado laboral. Además, las mujeres podrían requerir de mayores calificaciones o títulos que los hombres para insertarse en los mismos puestos de trabajo.

Respecto de la paridad de género de quienes rindieron la PSU y se matricularon en una universidad (en cualquier carrera), la Tabla 3 muestra que, en general, se mantienen las proporciones de género observadas. Más específicamente, respecto de los colegios de enseñanza media donde estudiaron quienes se inscriben para rendir la PSU 2018, la mayor la proporción de mujeres se encuentra en los colegios particulares 
subvencionados (54\%), lo que contrasta con los colegios particulares pagados, con sólo un $49.3 \%$. Esto sugiere una tendencia a que las familias privilegien estudios en colegios pagados por parte de hijos hombres, en detrimento de hijas mujeres. Sin embargo, la Tabla muestra también que son los colegios particulares pagados los que logran una clara mayor proporción de estudiantes que ingresan a las universidades (19.516 de 30.547). Aquí el nivel socioeconómico de las familias parece jugar un papel determinante.

En una perspectiva más amplia, la Figura 2 muestra la evolución de la proporción mujeres/hombres matriculados en alguna carrera universitaria chilena a través de los años. Puede observarse que el año 2011 se registró la menor proporción de mujeres (47\%), y desde entonces aparece una participación creciente de mujeres. El proceso de admisión 2016 marcó un cambio importante, ya que en ese año hay prácticamente paridad en la matrícula de hombres y mujeres (41.082 y 41.077 respectivamente).

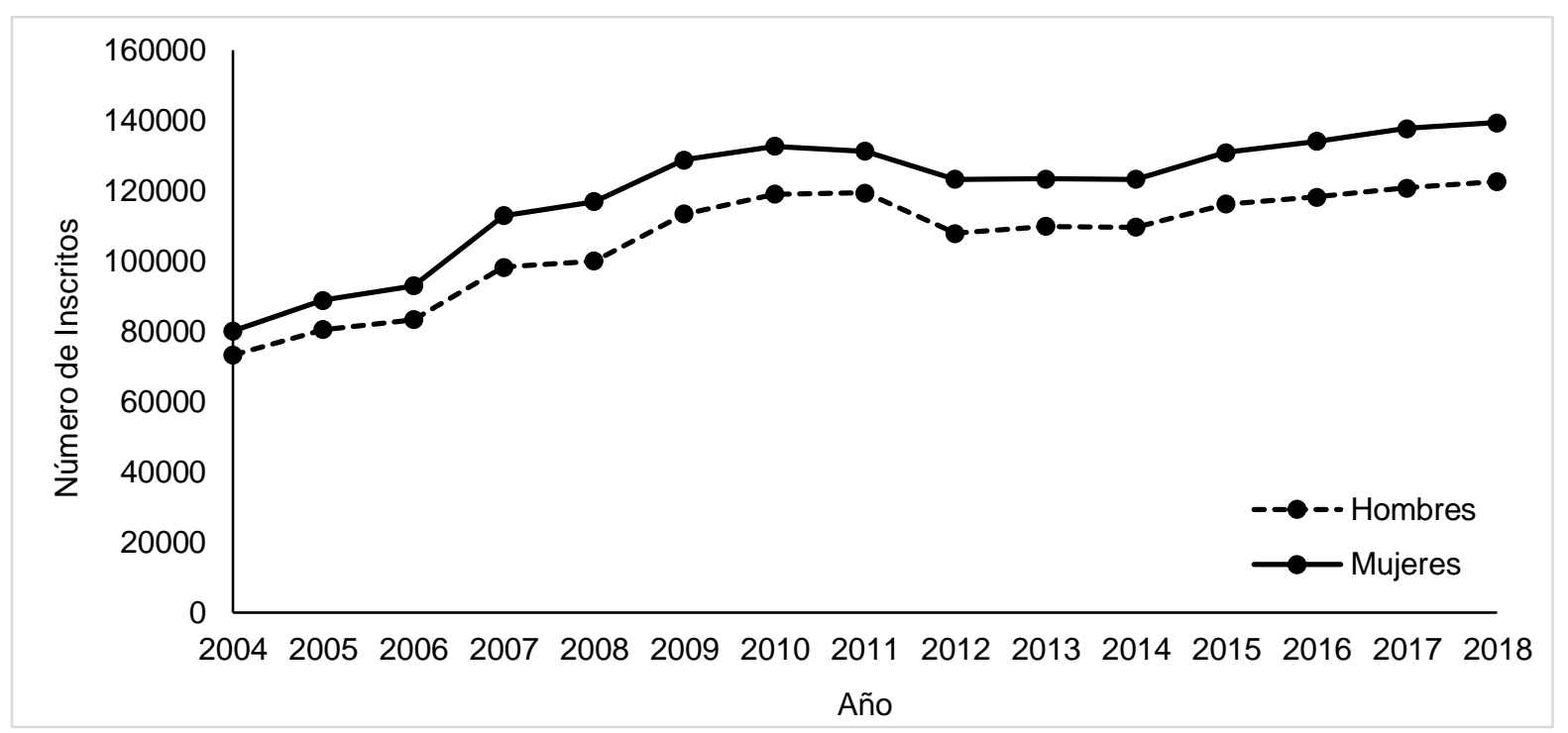

Fig. 1: Evolución del número de estudiantes inscritos para la PSU por sexo y año, periodo 2004-2018

Tabla 3: Tipo de establecimiento de egreso de enseñanza media y matrícula en universidades adscritas al SUA, año 2018.

\begin{tabular}{|l|l|l|l|l|}
\hline & \multicolumn{2}{|l|}{ Rindieron la PSU } & \multicolumn{2}{l|}{ Matriculados en alguna universidad } \\
\hline Tipo Establecimiento & Total & $\%$ Mujeres & Total & $\%$ Mujeres \\
\hline Particular Pagado & 30,547 & $49.3 \%$ & 19,516 & $49.5 \%$ \\
\hline Particular Subvencionado & 159,656 & $54.0 \%$ & 54,722 & $54.1 \%$ \\
\hline Municipal (Estatal) & 108,569 & $52.2 \%$ & 26,817 & $52.7 \%$ \\
\hline Totales & 298,772 & $52.9 \%$ & 101,055 & $52.8 \%$ \\
\hline
\end{tabular}

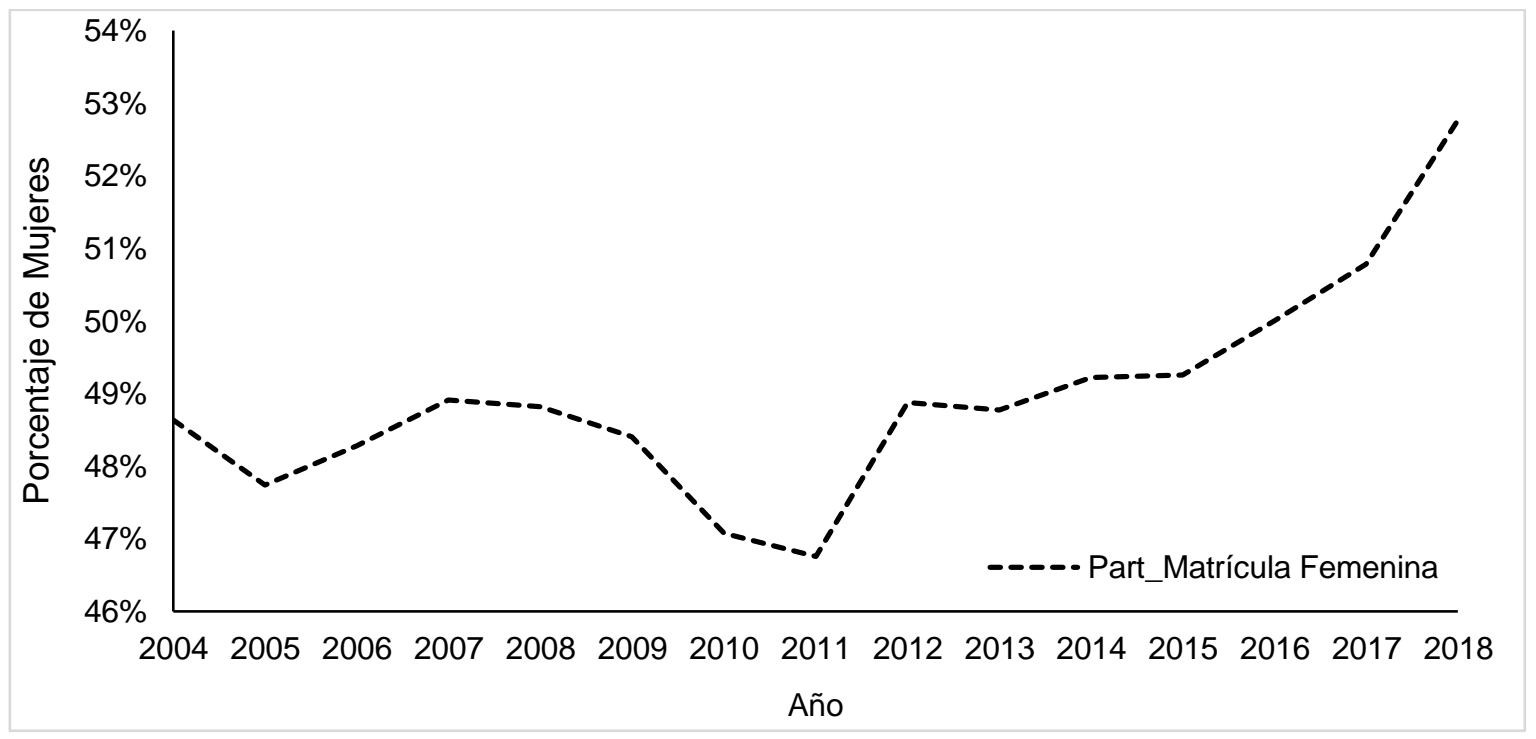

Fig. 2: Evolución de la proporción de mujeres matriculadas en universidades adscritas al SUA, periodo 2004-2018. Paridad en los resultados de la PSU 
Respecto de los resultados medidos por el puntaje promedio (lenguaje y matemáticas) en la PSU 2018, la Figura 3 muestra diferencias en los estudiantes de los tres tipos de colegios a favor de los hombres. También se aprecia claramente que los estudiantes de colegios particulares pagados logran los mejores puntajes promedio, y además que en ese tipo de colegios existe la menor diferencia entre hombres y mujeres, 9.12 puntos, en comparación con los 12.75 puntos de diferencia en colegios particulares subvencionados, y 20.33 puntos en los colegios municipales. Los resultados en este tipo de pruebas estandarizadas parecen reforzar los estereotipos género, las mujeres tienden a tener menos confianza, y se sienten menos capaces de estudiar carreras vinculadas a STEM (OECD, 2015; UNESCO, 2016). Esto coincide con los resultados de la prueba PISA 2015 para los países de la OECD, donde Chile es el tercer país con mayor brecha negativa hacia mujeres en ciencias y matemáticas (ComunidadMujer, 2017).

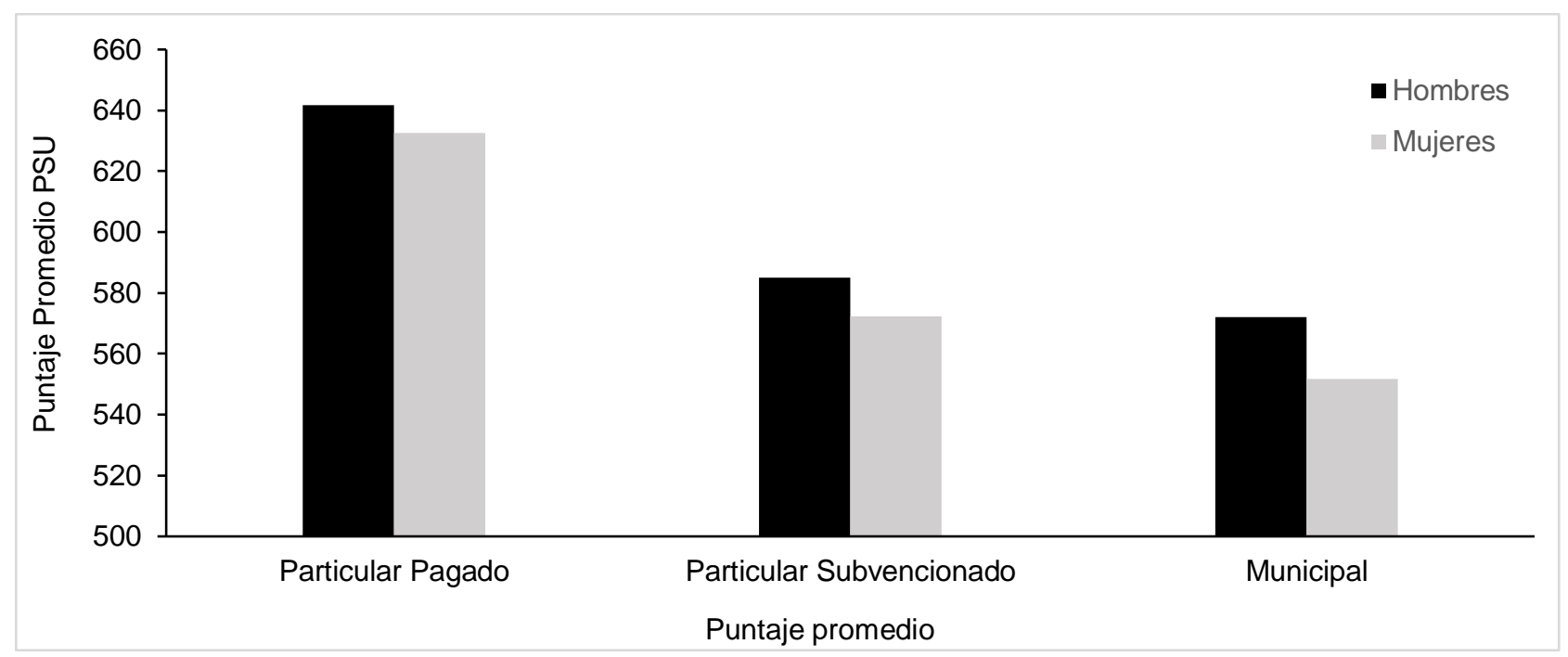

Fig. 3: Puntajes promedio PSU (lenguaje y matemáticas) de estudiantes que ingresan a alguna carrera en universidades adscritas al SUA, según sexo y tipo de colegio, año 2018

\section{Paridad en los matriculados}

Si bien las proporciones entre hombres y mujeres se mantienen hasta el ingreso a las universidades, es al momento de elegir una carrera del área de computación donde aparecen grandes brechas de género. En efecto, la Tabla 4 muestra que el año 2018 sólo el 19\% de los matriculados en carreras de esta área fueron mujeres. Adicionalmente, la Tabla muestra que hay una marcada preferencia de los estudiantes mujeres provenientes de colegios particulares pagados por este tipo de carreras. Esto se puede explicar en parte por los mayores puntajes promedio en la PSU que logran estos estudiantes. La Tabla muestra también que las mayores desigualdades de género en el área de computación se observan en estudiantes que provienen de colegios particular subvencionados.

Tabla 4: Matriculados en carreras del área de Computación en universidades adscritas al SUA, por tipo de establecimiento, Grupos 0 al 6 (año 2018).

\begin{tabular}{|l|l|l|}
\hline Tipo Establecimiento & Total de Matriculados en primer año de Carreras de Computación & $\%$ Mujeres \\
\hline Particular Pagado & 1,770 & $22.4 \%$ \\
\hline Particular Subvencionado & 2,396 & $17.1 \%$ \\
\hline Municipal (Estatal) & 1,215 & $18.8 \%$ \\
\hline Totales & 5,381 & $19.2 \%$ \\
\hline
\end{tabular}

El acceso a la tecnología parece ser clave, ya que está muy determinado por el nivel de ingresos de las familias (García, 2004). Blázquez et al., (2009) reporta que la labor motivacional de los profesores sería más influyente que la entregada en los hogares por parte de los familiares cercanos (fundamentalmente los padres). Fernández y Márquez (2019) también señalan que es clave que los profesores promuevan experiencias positivas en el aula y esto a su vez se relaciona con el proyecto educativo de los sostenedores y/o directores de los establecimientos educacionales. En consecuencia, la literatura recomienda un trabajo motivador en las aulas de los colegios para poder revertir la tendencia decreciente de las mujeres por preferir estudiar carreras del área tecnológica e ingeniería.

Esch (2005) señala que la escasez de modelos femeninos, la falta de motivación en las aulas y en el hogar, 
y los estereotipos hacia carreras de ciencias en ingeniería puede determinar la menor motivación de mujeres hacia estas disciplinas. Los estudios a nivel internacional muestran que el reclutamiento de mujeres en el área de computación e informática no se debe a la diferencia de habilidad o capacidad de ellas, sino que está relacionada con factores como la falta de modelos y de apoyo y una percepción de falta de autoestima y capacidad entre las mujeres (Lewis et al., 2007 y Stoilescu y Egodawatte, 2010)

En una perspectiva de largo plazo del periodo 2004-2018, aunque el número total de alumnos matriculados en los seis grupos de carreras ha experimentado incrementos importantes, la participación de mujeres ha disminuido en términos relativos. A continuación, analizamos la paridad de género en las matrículas de primer año para cada grupo de carreras (ver Tabla 2). En primer lugar, la Figura 4 muestra que, globalmente, la paridad de género ha tenido una leve tendencia hacia el empeoramiento. Observando más específicamente, las carreras del Grupo 1 y 2 son las que enfrentan una mayor caída en la participación femenina. Sin embargo, la Figura 4 muestra también que en el Grupo 0 (Ingeniería Civil plan común) se ha evidenciado un crecimiento considerable de la paridad, llegando al $27 \%$ de mujeres el año 2018. Una explicación de este fenómeno son las políticas que han surgido en este grupo de universidades. La Universidad de Chile creó el "Programa de Equidad de Género" para entregar 40 matrículas adicionales a mujeres a partir del año 2014. Este programa se hace cargo de las diferencias en educación y oportunidades, que reciben las mujeres en matemáticas y ciencias durante su formación en la enseñanza escolar. Este tipo de programas parecen ser importantes de impulsar en otras universidades, buscando ampliar la diversidad y cantidad de estudiantes mujeres en el área de las ciencias de la computación. Lo que está haciendo la Universidad de Chile con este programa es implementar acciones temporales para disminuir las desigualdades entre los sexos, en este caso, la matrícula de mujeres y hombres.

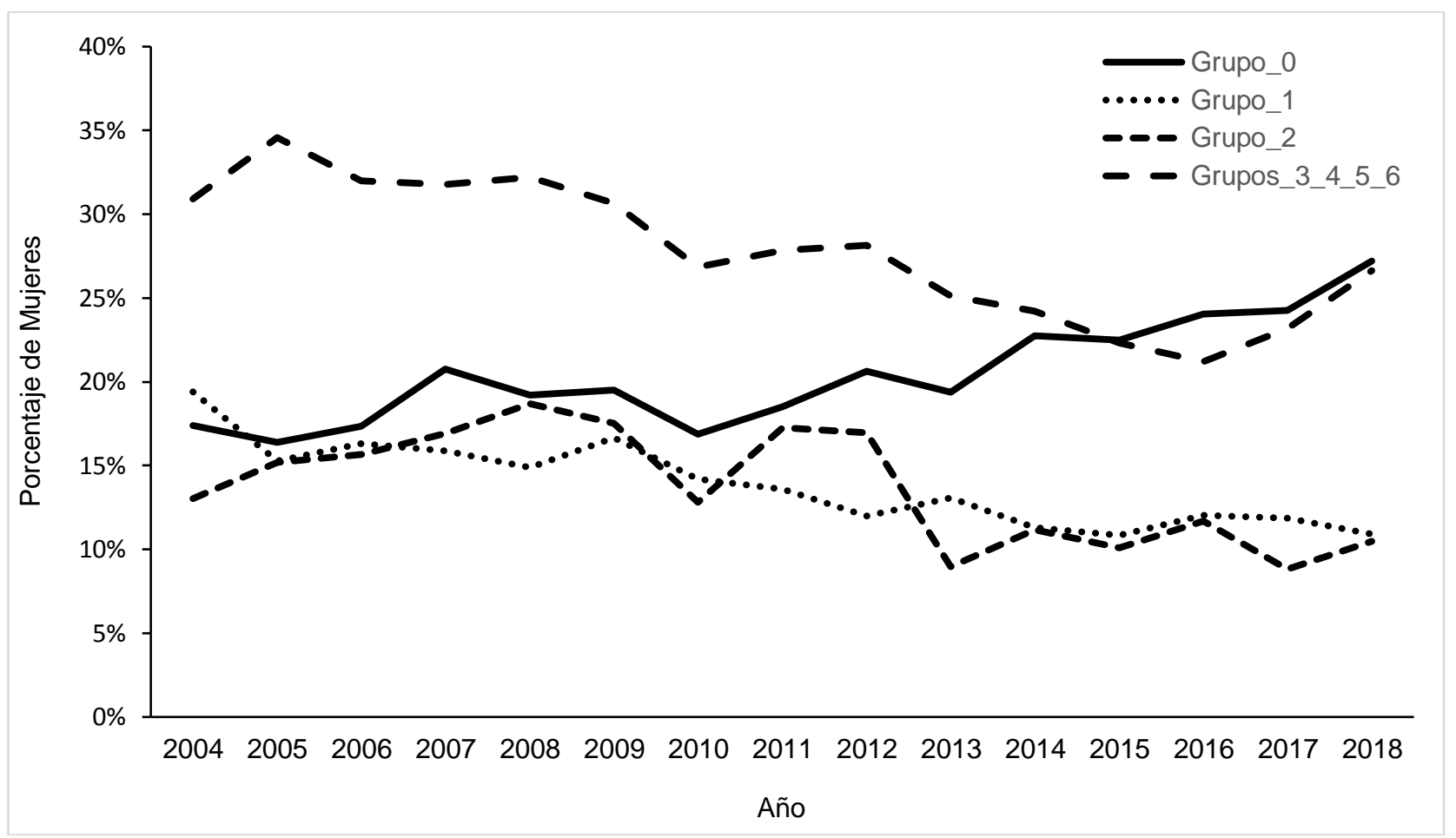

Fig. 4: Evolución de la participación de estudiantes mujeres matriculadas en carreras del área de computación e informática en universidades adscritas al SUA, por grupos de carreras

\section{Disposición de mujeres a estudiar carreras de computación en otras regiones}

Un último aspecto de interés se refiere a disposición para matricularse en carreras de computación en universidades en regiones distintas del domicilio. La Figura 5 muestra que, contrario a lo que podría pensarse, desde el año 2010 las mujeres han evidenciado mayor movilidad que los hombres. En particular, en el año 2004 sólo 129 mujeres se trasladaron, mientras que en 2018 lo hicieron 231 mujeres. En cambio, esta tendencia es inversa para los hombres, que pasó del 34\% (529) a un $29 \%$ (828). Este es un resultado relevante, ya que puede deducirse que cualquier universidad puede ofrecer programas de paridad de género, y la movilidad de las mujeres parece no ser una restricción para ser beneficiadas con este tipo de incentivos. Además, si consideramos que los talentos están igualmente distribuidos entre hombres y mujeres, las estrategias y políticas que generen mayor participación de la mujer en las carreras relacionadas con las ciencias de la computación pueden tener impactos significativos. 


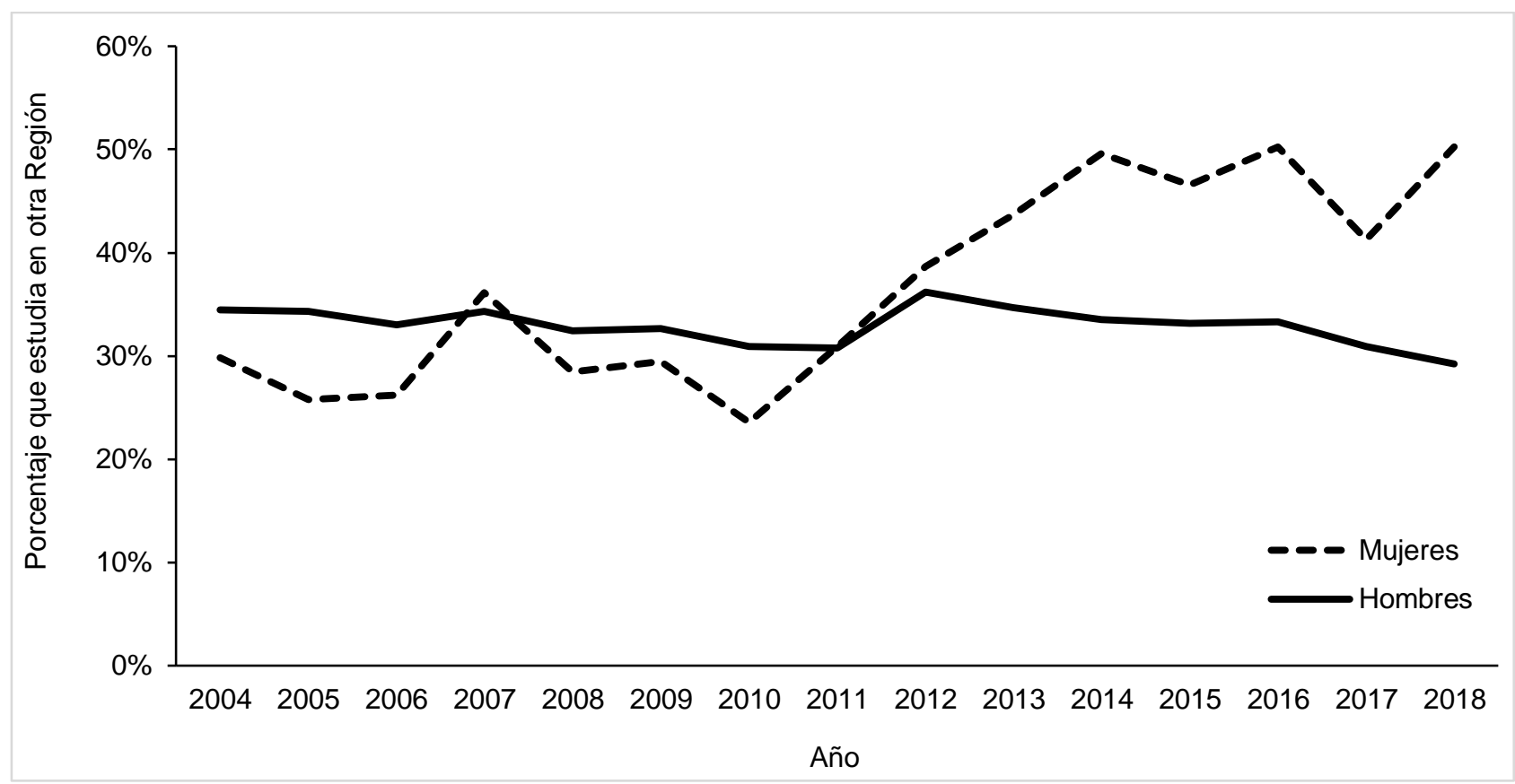

Fig. 5: Proporción de estudiantes matriculados en regiones distintas a su domicilio, en carreras de computación en universidades adscritas al SUA

\section{CONCLUSIONES}

De acuerdo al trabajo presentado y a los resultados obtenidos, se pueden plantear las siguientes conclusiones principales:

1.- Existe una mayor proporción de mujeres que de hombres que se inscriben y finalmente rinden la PSU en Chile. Esta proporción promedio en los últimos 15 años es de $53 \%$ de mujeres versus $47 \%$ de hombres.

2.- Hasta el año 2016 existió una mayor proporción de hombres matriculados en universidades adscritas al Sistema Único de Admisión (SUA), y a partir de ese año son más las mujeres quienes lo hacen.

3.- En las carreras de área de computación ofrecidas por las universidades adscritas al Sistema Único de Admisión (SUA) el año 2018 se matricularon sólo 5.381 mujeres, lo que representa sólo el $19.2 \%$ de total de matriculados en dichas carreras.

4.- Para el Grupo 0 de las carreras de Ingeniería Civil, plan común (acceso indirecto), ofrecidos por la U. de Chile, Pontificia U. Católica, UAI y U. de los Andes se evidencia un mejoramiento claro en la paridad de género, llegando a un ingreso del 27\% de mujeres en el año 2018.

5.- Para las carreras de computación del Grupo 1, ofrecidas por las demás universidades adscritas, la paridad de género se ha ido empeorando fuertemente en los años recientes, cayendo de $17 \%$ el año 2014 , al $11 \%$ en el año 2018.

6.- Los colegios particulares pagados son los que presentan una mayor proporción de mujeres matriculadas en las carreras del área de computación en el año 2018. Sin embargo, en la mayor cantidad de mujeres en términos absolutos a estas carreras proviene de los colegios particulares subvencionados.

7.- Las mujeres han evidenciado mayor movilidad que los hombres, matriculándose en carreras de computación en mayor proporción que los hombres en universidades de regiones diferentes al domicilio donde estudiaron su enseñanza media.

\section{REFERENCIAS}

Álvarez-Aguilar, N., González-Duéñez, V. y Castillo-Elizondo, J., Mujeres y Carreras de Ingeniería en la Universidad Autónoma de Nuevo León, en México: una Mirada desde las Vivencias de las Estudiantes, Formación Universitaria, 12(4), 85-94 (2019).

Anker, R., La Segregación Profesional entre Hombres y Mujeres. Repasos de las Teoría, Revista internacional del trabajo, 1 16(3). 343-370 (1997).

Arango, L., Género e Ingeniería: La Identidad Profesional en Discusión, Revista Colombiana de Antropología, 42, 129146 (2006). 
Blázquez, N., Flores, F. y Ríos, M. (Coords.), Investigación Feminista: Epistemología, Metodología y representaciones sociales (2 ${ }^{\mathrm{a}}$ Ed.), México DF: UNAM (2012).

Blázquez, C., Álvarez, P. y otros dos autores, Factores que Influencian la Motivación de Escolares por las Áreas Tecnológicas e Ingeniería, Calidad en la Educación, 31, 46-64 (2009).

ComunidadMujer, Mujer y trabajo: Brecha de Género en STEM, la Ausencia de Mujeres en Ingeniería y Matemáticas, Boletín 42, 15 (2017).

DEMRE., Bases de datos de las pruebas de selección universitaria (PSU) y procesos de postulación y selección a las universidades, Departamento de Evaluación, Medición y Registro Educacional (2019).

Downes, S. y Hobbs, V.J., An Exploratory Study of the Representation and Performance of Females in Information Technology at Murdoch University, Documento de Trabajo, School of Information Technology, Murdoch University (2000).

Esch, M., Career Building for Qualified Young Women Managers and Leaders in Science and Engineering: A public-private partnership initiative between technical universities and companies, Proceedings of the International Symposium on Women and ICT: Creating Global Transformation, 126. Baltimore, MD (2005).

Fernández, F. y Márquez, M., Participación de Mujeres en Carreras Informáticas en Chile: ¿Quiénes Eligen Informática Hoy?, ClioDinamica Consulting (2019).

García, P., El Género y las Ingenierías en Computación, Tarbiya (34) 81-89 (2004).

González, A.M., ¿Camuflaje o Transformación? Estrategia Profesional de las Mujeres en Carreras Tecnológicas Altamente Masculinizadas, Educar, 50(1), 187-205 (2014).

Hawes, G., y Corvalán, O., Competencias Fundamentales en Programas de Formación Profesional de Pregrado de la Universidad de Talca, Programa MECESUP TAL 0101, Universidad de Talca (2005).

Katz, S., Allbritton, D. y otros tres autores, Gender, achievement, and persistence in an undergraduate computer science program, ACM SIGMIS Bulletin, 37(4), 42-57 (2006).

Kesar, S., Closing the STEM Gap: Why STEM Classes and Careers Still Lack Girls and What We Can Do About It, Microsoft Philanthropies (2018).

Lewis, S., Lang, C. y McKay, J., An Inconvenient Truth: The Invisibility of Women in ICT. Australasian, Journal of Information Systems, 15(1) 59-76 (2007).

Machina, K. y Gokhale, A., Interventions for Increasing Male and Female Undergraduate Interest in Information Technology, Computers \& Education 87, 277-284 (2015).

Merelo, J.J. y Merelo, C., Evolución de la Matrícula Femenina en el Grado de Informática en Universidades Públicas Españolas, doi:10.13140/RG.2.2.19608.08969, Report number: GeNeura 2017-10-1, (2017).

Mora-Rivera, S., Coto-Chotto, M. y Villalobos-Murillo, J., Participación de las Mujeres en la Carrera de Ingeniería Informática de la Universidad Nacional y su Desempeño en los Cursos de Programación, doi: http://dx.doi.org/10.15359/ree.21-1.12, Revista Electrónica Educare, 21(1), 1- 22 (2017).

Mullally, S., Minority Rights and Gender Equality: Setting Limits to Collective Claims. International Journal on Minority and Group Rights 14, 263-283 (2007).

OECD., The ABC of Gender Equality in Education: Aptitude, Behavior, Confidence, PISA, OECD Publishing, Paris, https://doi.org/10.1787/9789264229945-en (2015).

Oliveros, M.A., Cabrera, E., Valdez, B., y Schorr, M., La Motivación de las Mujeres por las Carreras de Ingeniería y Tecnología, Entreciencias 4(9), 89-96 (2016).

Ordorika, I., Equidad de Género en la Educación Superior, Revista de la Educación Superior, 2 (174), 7-17 (2015).

Pappas, I.O., Aalberg, T., y otros tres autores, Gender Differences in Computer Science Education: Lessons learnt from an empirical study at NTNU, NIK 2016 conference (2016).

Pineda, E., y Gonzalez, C., Networking Skills in Latin America, White Paper, Cisco IDC Skills Gap (2016).

Rolando, R., Salamanca, J. y Aliaga, M., Evolución Matrícula Educación Superior de Chile, Periodo 1990 - 2009. SIES, División de Educación Superior, Ministerio de Educación de Chile (2010).

Stoilescu, D. y Egodawatte, G., Gender Differences in the Use of Computers, Programming, and Peer Interactions in Computer Science Classrooms, Computer Science Education, 20(4), 283-300 (2010).

UNESCO., Inequidad de Género en los Logros de Aprendizaje en Educación Primaria ¿Qué nos puede decir TERCE?, Oficina Regional de Educación para América Latina y el Caribe. OREALC/UNESCO, Santiago, Chile (2016).

Vilner, T. y Zur, E., Once she makes it, she is there: Gender Differences in Computer Science Study, ACM SIGCSE Bulletin, 38(3), 227-231 (2006).

Williams, F., y Emerson, C., Becoming Leaders: A Handbook for Women in Science, Engineering and Technology, St. John's, NL: Canada Memorial University of Newfoundland (2002). 
